\title{
IL REGOLAMENTO (UE) N. 1259/2010 SULLA LEGGE APPLICABILE AL DIVORZIO E ALLA SEPARAZIONE PERSONALE NELLA RECENTE PRASSI GIURISPRUDENZIALE ITALIANA
}

\author{
RECENT ITALIAN CASE-LAW CONCERNING (EU) \\ REGULATION ON THE LAW APPLICABLE TO DIVORCE \\ AND LEGAL SEPARATION
}

\author{
FEDERICA FALCONI \\ Ricercatore di Diritto internazionale \\ Università degli Studi di Pavia
}

Recibido: 15.01.2018 / Aceptado: 02.01.2018

DOI: https://doi.org/10.20318/cdt.2018.4138

\begin{abstract}
Riassunto: Il presente contributo propone una breve analisi della prassi applicativa italiana in relazione al regolamento (UE) n. 1259/2010 in tema di legge applicabile al divorzio e alla separazione personale. Solo in un ristretto numero di casi le parti si sono avvalse della facoltà di optio legis loro concessa dall'art. 5 del regolamento, accordando preferenza alla legge nazionale comune. Più spesso, in mancanza di un accordo delle parti, la legge applicabile è individuata in applicazione dell'art. 8: ciò conduce nella maggior parte delle ipotesi all'applicazione della legge dello Stato di residenza abituale dei coniugi, con il risultato di favorire l'integrazione sociale e ripristinando altresì la corrispondenza tra forum e jus.

Parole chiave: Regolamento (UE) n. 1259/2010, divorzio e separazione personale, conflitti di leggi, diritto internazionale privato dell'Unione europea, optio legis, legge applicabile in mancanza di scelta.

Abstract: This article offers a brief analysis of the Italian case-law concerning Regulation (EU) No $1259 / 2010$ on the law applicable to divorce and legal separation. Only in a few cases, spouses have chosen the applicable law according to Article 5, by designating the law of their State of nationality. More frequently, absent a valid choice by the spouses, the law applicable to divorce or legal separation has been determined in accordance with Article 8: this usually leads to the application of the law of the country where the spouses are habitually resident, thereby promoting social integration and also restoring the correspondence between forum and jus.

Keywords: Regulation (EU) No 1259/2010, divorce and legal separation, conflict-of-laws rules, private international law of the European Union, choice of law agreement, applicable law in the absence of a choice by the parties.
\end{abstract}

Sommario: I. Cenni introduttivi. II. La prassi applicativa sull'art. 5 del regolamento (UE) n. 1259/2010: l'optio legis. III. La prassi applicativa sull'art. 8 del regolamento (UE) n. 1259/2010: l'individuazione della legge applicabile in caso di mancato accordo tra i coniugi. IV. Osservazioni conclusive. 


\section{Cenni introduttivi}

1. Come noto, il regolamento (UE) n. 1259/2010, che attua una cooperazione rafforzata in materia di legge applicabile al divorzio e alla separazione personale, conferisce ai coniugi la facoltà di designare la legge applicabile, quantunque entro un novero di opzioni tassativamente prestabilite ${ }^{1}$. In particolare, secondo quanto disposto dall'art. 5, par. 1, la scelta dei coniugi può avere ad oggetto: a) la legge dello Stato di residenza abituale dei coniugi; $b$ ) la legge dello Stato di ultima residenza abituale dei coniugi ${ }^{2}$, purché uno dei due vi risieda ancora al momento della scelta; $c$ ) la legge dello Stato di cittadinanza dei coniugi ovvero di uno soltanto di essi e, infine, $d$ ) la legge del foro. In tutti i casi, si tratta di leggi che esprimono una connessione più o meno significativa con la fattispecie da regolare ${ }^{3}$. Ciò vale, almeno in linea di principio, anche per la lex fori, da individuarsi alla stregua dei criteri stabiliti dal regolamento (CE) n. 2201/2003 (Bruxelles II-bis), ispirati alle medesime esigenze di prossimità ${ }^{4}$ Va detto, peraltro, che l'inclusione della lex fori nel ventaglio delle leggi designabili - nonché la sua contestuale previsione a titolo di criterio oggettivo - trova ragion d'essere in considerazioni diverse da quelle appena evocate: dalla ripristinata corrispondenza tra forum e ius, infatti, discendono effetti vantaggiosi che non si esauriscono in una mera semplificazione del compito dell'organo giudicante e che possono apprezzarsi in special modo sotto il profilo dell'efficienza processuale e della tutela dei diritti, considerato come i profili processuali risultino in questo settore del diritto strettamente interconnessi a quelli sostanziali ${ }^{5}$.

Il ruolo di primaria importanza attribuito alla concorde volontà dei coniugi nella determinazione della lex divortii rappresenta senza dubbio l'innovazione più significativa introdotta dal regolamento ed invero, prima della sua entrata in vigore, soltanto una ristretta minoranza tra gli Stati membri riconosceva ai coniugi la facoltà di esercitare l'optio legis in questa materia ${ }^{6}$. Varie e molteplici sono le ragioni di tale "rivoluzionaria" apertura, per quanto limitata ai soli Stati membri che, come l'Italia, hanno scelto di partecipare alla cooperazione rafforzata ${ }^{7}$ : dall'esigenza di garantire la certezza del diritto e la prevedibilità del diritto applicabile - secondo una linea di tendenza comune a tutti gli altri regolamenti dove l'optio legis è parimenti accolta, anche se con modulazioni differenti - a quella di consentire alla coppia

${ }^{1}$ Regolamento (UE) n. 1259/2010 del Consiglio, del 20 dicembre 2010, relativo all'attuazione di una cooperazione rafforzata nel settore del diritto applicabile al divorzio e alla separazione personale, GUUE L 343 del 29 dicembre 2010, p. $10 \mathrm{ss}$. Nel testo, il regolamento sarà indicato anche come "Roma III". Attualmente, sono diciassette gli Stati membri che hanno aderito alla cooperazione rafforzata e risultano perciò vincolati all'applicazione della normativa uniforme (Austria, Belgio, Bulgaria, Francia, Germania, Italia, Lettonia, Lussemburgo, Malta, Portogallo, Romania, Slovenia, Spagna, Ungheria, Lituania, Grecia ed Estonia).

${ }^{2}$ Il riferimento è da intendersi alla residenza abituale dei coniugi in uno stesso Stato, senza che sia necessario che si tratti di una residenza comune. Questo significa che i coniugi che, seppur separatamente vivono nel medesimo Stato, come invero spesso accade quando viene introdotta l'istanza di separazione o di divorzio, possono scegliere la legge di tale Stato.

${ }^{3}$ L'esigenza che la scelta dei coniugi cada sulla legge di uno Stato con il quale sussiste un "legame particolare" è espressa dal considerando n. 16. Analoghe esigenze di prossimità ispirano i criteri dettati all'art. 8 per le ipotesi, come si dirà frequenti nella prassi, di mancata scelta.

4 Regolamento (CE) n. 2201/2003 del Consiglio, del 27 novembre 2003, relativo alla competenza, al riconoscimento e all'esecuzione delle decisioni in materia matrimoniale e in materia di responsabilità genitoriale, che abroga il regolamento (CE) n. 1347/2000, in GUUE L 338 del 23 dicembre 2003, p. 1 ss. Sul punto, v. la Relazione esplicativa redatta dalla Prof. Alegría Borrás relativa alla Convenzione del 28 maggio 1998, concernente la competenza, il riconoscimento e l'esecuzione delle decisioni nelle cause matrimoniali, in GUCE, C 221/37, p. 27 ss., par. 30.

5 Rilevano tale interconnessione R. CLERICI, "Il nuovo regolamento dell'Unione europea sulla legge applicabile al divorzio e alla separazione personale", Famiglia e diritto, 2011, p. 1053 ss.; P. Franzina, "The Law Applicable to Divorce and Legal Separation under Regulation (EU) No 1259/2010", questa Rivista, 2011, p. 85 ss.

${ }^{6}$ Anche se entro margini generalmente più ristretti rispetto a quelli della disciplina uniforme oggi in vigore, l'optio legis era ammessa soltanto da Germania, Belgio e Paesi Bassi: sul punto, I. VIAREnGo, "Il regolamento UE sulla legge applicabile alla separazione e al divorzio e il criterio della volontà delle parti", Rivista di diritto internazionale privato e processuale, 2011, p. 601 ss., p. 611, nota 32. Per quanto riguarda l'Italia, rispetto ai procedimenti introdotti prima del 21 giugno 2012, la materia continua ad essere disciplinata dall'art. 31 della legge 31 maggio 1995, n. 218 ("Riforma del diritto internazionale privato italiano"), a mente del quale la separazione e lo scioglimento del matrimonio sono regolati dalla legge nazionale comune dei coniugi al momento della domanda e, in mancanza (ovvero in presenza di più leggi nazionali comuni), dalla legge dello Stato in cui è prevalentemente localizzata la vita matrimoniale, salva in entrambi i casi l'operatività del rinvio nei limiti definiti dall'art. 13 della stessa legge.

7 Il carattere rivoluzionario del regolamento, per lo spazio concesso all'autonomia dei coniugi, è sottolineato da R. CLERICI, "Il nuovo regolamento dell'Unione europea sulla legge applicabile al divorzio e alla separazione personale" cit., p. 1054. 
di accedere più agevolmente al divorzio (favor divortii), selezionando la legge più confacente alle proprie esigenze, sul presupposto che ciò risulti funzionale a garantire l'esercizio effettivo della libertà di circolazione delle persone nello spazio europeo ${ }^{8}$. Oltre a ciò, occorre notare che la volontà dei coniugi, così come disciplinata dall'art. 5 , si intende diretta a selezionare le disposizioni di diritto materiale dell'ordinamento richiamato, ad esclusione delle disposizioni di diritto internazionale privato, stante l'univoca esclusione del rinvio disposta dall'art. 11 dello stesso regolamento.

2. Vi è poi un ulteriore aspetto nel quale si riflette la portata innovativa del regolamento (UE) $n$. 1259/2010, il quale, nel concedere spazio all'autonomia internazionalprivatistica dei coniugi nei termini sopra brevemente accennati, introduce al contempo il concetto di "scelta informata" con l'obiettivo di garantire che ciascun coniuge sia consapevole delle conseguenze giuridiche e sociali derivanti dall'accordo raggiunto 9 . In prima battuta, si richiede che, prima di procedere alla designazione della legge applicabile, i coniugi abbiano accesso ad "informazioni aggiornate relative agli aspetti essenziali della legge nazionale e dell'Unione e delle procedure in materia di divorzio e di separazione personale" ${ }^{\text {" }}$. L'optio legis viene quindi sottoposta al rispetto di precisi requisiti formali: in base all'art. 7, par. 1, l'accordo deve essere come minimo "redatto per iscritto, datato e firmato da entrambi i coniugi", con ciò escludendo che la volontà possa essere manifestata tacitamente. Gli stessi giudici sono chiamati a svolgere un compito di primaria importanza affinché il principio della scelta informata venga concretamente rispettato e non resti una mera enunciazione di principio.

3. Volgendo lo sguardo alla prassi applicativa italiana, e circoscrivendo l'oggetto di questa breve rassegna ad alcune delle principali pronunce emanate nel biennio 2016-2017, se ne trae l'impressione che, in apparente controtendenza rispetto ai primi anni di applicazione del regolamento ${ }^{11}$, $\mathrm{i}$ casi in cui i coniugi hanno esercitato l'optio legis costituiscano una minoranza ${ }^{12}$. Questo dato, come si dirà meglio in seguito analizzando i criteri oggettivi previsti dall'art. 8 del regolamento in relazione alle ipotesi di mancata scelta, non appare del tutto casuale: con l'insorgere della crisi matrimoniale, infatti,

${ }^{8}$ Ampiamente sul ruolo della lex voluntatis nel regolamento Roma III e sulle opzioni di politica legislativa che la sottendono, cfr. A.-L. Calvo Caravaca, J. Carrascosa González, "La Ley aplicable al divorcio en Europa: el futuro Reglamento Roma III", questa Rivista, 2009, p. 36 ss., p. 52 ss.; P. FranzINA, "L'autonomia della volontà nel regolamento sui conflitti di leggi in materia di separazione e divorzio", Rivista di diritto internazionale, 2011, p. 488 ss.; I. QueIrolo, L. CARPANETO, "Considerazioni critiche sull'estensione dell'autonomia privata a separazione e divorzio nel regolamento "Roma III"', Rivista di diritto internazionale privato processuale, 2012, p. 56 ss. In un'ottica di raffronto rispetto alla disciplina dell'optio legis in materia di obbligazioni contrattuali, v. R. CLERICI, "Il ruolo dell'autonomia privata tra espansione e limiti di operatività nel regolamento (UE) n. 1259/2010 sulla legge applicabile al divorzio e alla separazione personale", Diritto del commercio internazionale, 2012, p. 351 ss.; J. CARrascosa GonzÁlez, F. SeAtzu, "La legge applicabile al divorzio e alla separazione personale dei coniugi e al divorzio nella proposta di regolamento Roma III", Studi sull'integrazione europea, 2010, p . 49 ss. Infine, per un'analisi delle diverse clausole, previste dal regolamento Roma III, suscettibili di condurre alla disapplicazione della lex causae, v. J. CARRASCOSA GonZÁLEZ, "Esclusione dell'applicazione della legge regolatrice del divorzio e regolamento Roma III", ibidem, 2017, p. 267 ss.

9 Come risulta espressamente dal considerando n. 18, la scelta informata di entrambi i coniugi rappresenta un "principio essenziale" del regolamento. Tale principio trova corrispondenza nei recenti regolamenti (UE) n. 1103 e 1104, del 24 giugno 2016, che attuano una cooperazione rafforzata in materia di giurisdizione, legge applicabile, riconoscimento ed esecuzione delle decisioni per quanto riguarda rispettivamente i regimi patrimoniali tra coniugi e gli effetti patrimoniali delle unioni registrate, in GUUE L 183 dell'8 luglio 2016, p. 1 ss.

10 A tal fine, come sottolinea il considerando $\mathrm{n} .17$, i coniugi possono avvalersi del sistema di informazione on line, regolarmente aggiornato dalla Commissione, istituito con la decisione 2001/470/CE, del 28 maggio 2001, relativa all'istituzione di una rete giudiziaria europea in materia civile e commerciale, in GUCE L 174, 27 giugno 2001, p. 10 ss.

11 Sulla prassi anteriore al periodo qui considerato, cfr. C. CAMPIGLIO, "Prime applicazioni delle norme europee sui divorzi 'internazionali'”, Nuova giurisprudenza civile commentata, 2015, p. 173 ss.; I. VIARENGO, "International Divorce Proceedings in Italy: Issues Arising in the Case Law", Rivista di diritto internazionale privato e processuale, 2016, p. 700 ss.; ID., "Rapporto sull'applicazione del regolamento (UE) n. 1259/2010 del 20 dicembre 2010 relativo all'attuazione di una cooperazione rafforzata nel settore della legge applicabile al divorzio e alla separazione personale ("Roma III")", in S. Bariatti, I. Viarengo, F. C. Villata (a cura di), La giurisprudenza italiana sui regolamenti europei in materia civile e commerciale e di famiglia, Padova, Cedam, 2016, p. 397 ss.

12 Salvo diversa indicazione, le sentenze citate nel testo sono per lo più inedite e sono state attinte dal database pubblico del Progetto "Planning The Future of Cross Border Families: A Path through Coordination - 'EUFam's", www.eufams.unimi. it (la versione definitiva è aggiornata al 28 dicembre 2017), dove ciascuna è corredata da una breve, ma efficace sintesi dei fatti dedotti in giudizio e dei profili di diritto affrontati. 
l'individuazione della lex divortii si somma ai vari e molteplici aspetti suscettibili di costituire oggetto di contesa tra i coniugi. In altri casi, sarà invece l'irreperibilità di uno di essi a determinare l'impossibilità di acquisirne materialmente il consenso.

Quando invece i coniugi si sono avvalsi di tale facoltà, la scelta è caduta pressoché invariabilmente sulla legge di uno Stato non membro dell'Unione europea, la cui legislazione consente di accedere al divorzio senza la necessità di un previo periodo di separazione o comunque in modo più spedito, con un minor dispendio di risorse economiche ${ }^{13}$. Peraltro, l'opzione a favore della legge nazionale può spiegarsi anche in un'ottica diversa, in particolare allorché i coniugi abbiano interesse a conservare intatte le tradizioni culturali vigenti nel proprio Paese di origine a prescindere da quello di residenza ${ }^{14}$.

In prevalenza, si noti, i casi analizzati riguardano coppie di stranieri integrate da tempo nella realtà sociale italiana, dopo aver qui stabilito la propria residenza abituale ${ }^{15}$.

\section{La prassi applicativa sull'art. 5 del regolamento (UE) n. 1259/2010: l'optio legis}

4. Le decisioni che hanno pronunciato il divorzio in applicazione della legge designata dagli stessi coniugi ai sensi dell'art. 5 del regolamento (UE) n. 1259/2010 si sono concentrate in prevalenza sui profili riguardanti le modalità e i tempi di manifestazione della scelta. Tra queste, merita considerazione quella emessa dal Tribunale di Belluno il 27 ottobre $2016^{16}$. Il caso riguarda una coppia di cittadini albanesi, trasferitisi in Italia dopo essersi sposati in patria. Di comune accordo, i coniugi chiedono lo scioglimento del matrimonio senza previa separazione, così come consentito dalla legge albanese espressamente invocata nel ricorso introduttivo.

Dopo aver verificato positivamente la sussistenza della giurisdizione italiana in base all'art. 3, par. 1, lett. a), primo trattino, del regolamento Bruxelles II-bis (residenza abituale dei coniugi) ${ }^{17}$, il Tribunale ritiene applicabile l'art. 5, par. 1, lett. c) del regolamento Roma III e ciò a dispetto del fatto che la legge albanese sia invocata dalle parti "nella convinzione dell'operatività dell'art. $31,1^{\circ}$ comma, 1 . 31 maggio 1995 n. 218".

Al riguardo, il Tribunale rileva come l'accordo si perfezioni con la designazione della medesima legge da parte dei coniugi "indipendentemente dalle ragioni di tale concorde indicazione", ossia, nella fattispecie dedotta in giudizio, a prescindere dal fatto che la legge albanese sia invocata dalle parti sulla base di un erroneo convincimento, ritenendo pertanto sufficiente la concorde indicazione ad opera dei coniugi del Codice della famiglia della Repubblica albanese: la legge dello Stato di cittadinanza

13 Identifica tale tendenza sin dalle prime applicazioni del regolamento C. CAMPIGLIO, op. cit., p. 176.

14 Per tale rilievo, v. I. Viarengo, "Rapporto sull'applicazione del regolamento (UE) n. 1259/2010...”, cit., p. 399, che osserva inoltre come l'applicazione della legge nazionale contribuisca ad agevolare il riconoscimento della sentenza di divorzio nello Stato di origine.

15 Tra le molteplici circostanze idonee in concreto a conferire alla fattispecie da regolare carattere "transnazionale", che come previsto espressamente dall'art. 1, par. 1, del regolamento, costituisce il presupposto per l'applicazione della disciplina uniforme, assume rilievo anche la semplice residenza all'estero di uno dei due coniugi, aventi la medesima nazionalità: sul punto, v. il Libro verde della Commissione sulla legge applicabile e sulla giurisdizione in materia di divorzio del 14 marzo 2005, COM (2005) 82 def.

16 Il testo è pubblicato in Rivista di diritto internazionale privato processuale, 2017, p. 755 . Lo stesso Tribunale, con sentenza del 23 dicembre 2014, in Rivista di diritto internazionale privato processuale, 2016, p. 207 ss., in relazione alla richiesta di scioglimento del matrimonio celebrato in Italia da due cittadini tunisini, ha ritenuto valida l'optio legis dei coniugi a favore della legge tunisina, invocata dai coniugi - rispettivamente nel ricorso e nella comparsa di costituzione - sebbene tale legge fosse stata ricondotta erroneamente all'art. 31 della legge n. 218/1995. Oltre a quelle citate nel testo, hanno dato applicazione alla legge prescelta dalle parti in base all'art. 5 del regolamento: TRIB. RAVENNA, 31 marzo 2016 (con applicazione della legge marocchina, quale legge nazionale comune dei coniugi); TRIB. RomA, 12 aprile 2016 (con applicazione della legge rumena, in quanto legge nazionale comune dei coniugi); TRIB. PADOVA, 25 luglio 2016 (la scelta è caduta sulla legge rumena, essendo la moglie rumena e il marito italiano); TriB. BERgamo, 25 ottobre 2016 (con applicazione della legge cinese, legge nazionale comune); TriB. PADOVA, 8 settembre 2017 (con applicazione della legge marocchina, quale legge nazionale comune).

17 A tal fine, in linea con la nozione autonoma elaborata dalla Corte di Giustizia, il Tribunale accerta che al momento della proposizione della domanda "le parti avevano effettivamente fissato, con carattere di stabilità, il centro principale e permanente dei propri interessi e relazioni in provincia di Belluno, quale luogo del concreto e continuativo svolgimento della vita personale e lavorativa, alla data di proposizione della domanda giudiziale". 
al momento della conclusione dell'accordo - in questo caso comune, ma in ipotesi anche di uno dei coniugi soltanto - figura infatti tra quelle che le parti possono designare sulla base all'art. 5, par. 1, del regolamento.

5. Passando quindi a verificare la validità della scelta contenuta nel ricorso congiuntamente sottoscritto dalle parti, il Tribunale ritiene soddisfatti sia i requisiti temporali, sia i requisiti formali minimi indicati dallo stesso regolamento. Quanto ai requisiti temporali, la designazione della legge albanese risulta effettuata in termini univoci sin dal ricorso introduttivo del giudizio, in linea con l'art. 5 , par. 2, del regolamento, che fissa al momento in cui è adita l'autorità il termine ultimo entro il quale le parti possono concludere l'accordo, salvo il temperamento di cui al par. 3, per cui l'optio legis può essere esercitata in corso di causa se la legge del foro lo consente ${ }^{18}$. Quanto invece ai requisiti formali, il Tribunale reputa l'espresso richiamo alla legge albanese operato nel ricorso congiuntamente sottoscritto dai coniugi senz'altro idoneo a soddisfare le forme minime richieste dall'art. 7, par. 1, rilevando come in proposito l'Italia non abbia ritenuto necessario prevedere requisiti di forma più rigorosi ${ }^{19}$.

6. In merito alla consapevolezza della scelta effettuata dai coniugi, il Tribunale osserva come "dall'esame degli atti del procedimento emerge in modo chiaro e preciso come le parti abbiano formulato l'indicazione della legge albanese sul presupposto della possibilità di ottenere l'immediato scioglimento del matrimonio, senza la preventiva separazione personale, diversamente da quanto richiesto dalla legge italiana", la cui applicazione, ove non si ritenesse validamente formato l'accordo fra le parti, discenderebbe dall'art. 8, lett. a), del regolamento ${ }^{20}$. Quest'ultimo passaggio appare particolarmente importante nell'iter argomentativo seguito dal Tribunale. Infatti, nell'escludere che l'errata convinzione circa l'operatività della disciplina di conflitto interna sia tale da inficiare la scelta effettuata dalle parti a favore della legge albanese, ciò che appare dirimente nel senso di ritenere soddisfatto il requisito della scelta informata è il riferimento espresso operato dai coniugi nel ricorso introduttivo al contenuto specifico della legge albanese, ritenuto dagli stessi coniugi maggiormente idoneo a soddisfare le proprie esigenze rispetto a quanto previsto dalla legge italiana ${ }^{21}$.

7. Correttamente, infine, il Tribunale esclude siano rinvenibili altri elementi per escludere l'operatività della legge albanese, prescelta dalle parti: non il fatto che si tratti della legge di uno Stato non membro dell'Unione europea, dato che per espressa disposizione normativa le norme uniformi

18 Nell'ordinamento italiano non si rinviene una precisa disposizione normativa in merito a tale aspetto. Nondimeno, la giurisprudenza ha affermato il principio secondo il quale l'optio legis, da qualificarsi come negozio di diritto processuale, può avvenire fino al momento in cui alle parti è consentito integrare le proprie domande (art. 709, comma 3, cod. proc. civ.), momento che - in caso di trasformazione del giudizio da contenzioso a consensuale - coincide con l'udienza di precisazione delle conclusioni.

19 È in particolare dall'art. 7, par. 2, che eventuali requisiti di forma supplementari previsti dall'Italia, quale Stato di residenza abituale dei coniugi, si vedrebbero conferito rilievo.

${ }^{20}$ Più precisamente, dall'art. 8 , lett. $a$ ), che prevede la legge della residenza comune dei coniugi al primo posto di un'articolata sequenza di criteri.

21 Ad una diversa conclusione era giunto, con ordinanza datata 11 dicembre 2012, il Tribunale di Milano, in Rivista di diritto internazionale privato processuale, 2013, p. 753. Anche in questo caso, i coniugi si erano espressamente richiamati negli scritti difensivi alla propria legge nazionale (in particolare, al codice civile dell'Ecuador) sulla base della convinzione che il criterio oggettivo della cittadinanza dei coniugi quale previsto dall'art. 31, $1^{\circ}$ comma, della legge 218/1995 fosse in effetti "l'unico disponibile per disciplinare la fine del matrimonio e le pronunce conseguenti". Secondo i giudici milanesi, le parti non avevano usufruito della facoltà di scelta offerta dal regolamento (UE) n. 1259/2010 poiché "la legge ritenuta applicabile era già pre-impostata dal disposto dell'art. $31,1^{\circ}$ comma, 1. 218/1995 sulla base del quale nessuna opzione era possibile". Partendo da questo presupposto, il Tribunale rileva peraltro come la designazione della legge applicabile può essere effettuata dai coniugi anche nel corso del giudizio, se ciò è ammesso dalla lex fori (art. 5, par. 3, regolamento Roma III) ed invoca l'art. 709 cod. proc. civ. - secondo il quale, con l'ordinanza di fissazione dell'udienza di comparizione delle parti, il Presidente assegna un termine al ricorrente e al convenuto per il deposito di memorie in cui indicare, tra gli altri, gli elementi di diritto costituenti le ragioni della domanda e, per il convenuto, le relative eccezioni processuali e di merito non rilevabili d'ufficio - per affermare la possibilità per il Presidente di informare le parti sulla facoltà di esercitare l'optio legis. Ciò puntualmente avviene nel caso di specie, con un preciso invito formulato alle parti dal Presidente ad indicare, nella memoria integrativa e nell'atto di costituzione, la legge che intendono sia applicata nel giudizio, in particolare manifestando la volontà che sia applicata la legge ecuadoriana (legge dello Stato di comune cittadinanza) ovvero quella italiana (legge dello Stato di residenza abituale). 
hanno carattere universale o erga omnes (art. 4) e sono pertanto suscettibili di condurre all'applicazione della legge di Stati terzi, oltre che di Stati membri che non partecipano alla cooperazione rafforzata, e nemmeno l'assenza di una precedente sentenza di separazione. In merito a tale ultimo aspetto, infatti, il Tribunale esclude possa configurarsi un contrasto con l'ordine pubblico, secondo quanto previsto dall'art. 12 del regolamento (UE) n. 1259/2010, ritenendo sufficiente ai fini della pronuncia di divorzio la comprovata impossibilità di ricostruire la comunione spirituale e materiale tra i coniugi ${ }^{22}$.

8. Si riferisce a fatti in gran parte analoghi, ma sembra comunque meritevole di attenzione, la sentenza pronunciata dal Tribunale di Treviso in data 20 settembre $2016^{23}$. Si tratta, come nella fattispecie analizzata in precedenza, di una coppia di coniugi stranieri - entrambi di nazionalità moldava - inserita stabilmente nella realtà sociale italiana. In questo caso, però, il procedimento ha natura contenziosa: l'istanza di divorzio viene presentata dalla moglie invocando espressamente nel ricorso introduttivo il Codice della Famiglia moldavo, il quale consente ai coniugi di ottenere lo scioglimento del vincolo coniugale senza previa separazione. Costituitosi in giudizio, il marito non contesta l'applicazione della legge moldava né si oppone alla domanda di divorzio, limitandosi a chiedere il rigetto della domanda di addebito formulata dalla moglie.

9. Come risulta dall'ordinanza di rimessione della causa sul ruolo istruttorio, l'applicabilità della legge moldava viene inizialmente revocata in dubbio dal Tribunale, non risultando in tal senso alcun accordo tra i coniugi. Veniva perciò evocata l'applicazione della legge italiana sulla base di quanto previsto dall'art. 8, lett. $a$ ), del regolamento Roma I, quale legge dello Stato di residenza comune dei coniugi, prospettando una declaratoria di ammissibilità del ricorso di divorzio - nonché delle ulteriori domande formulate in merito all'affidamento e al mantenimento della prole - posto che l'ordinamento italiano ammette che sia pronunciato il divorzio, eccetto che in circostanze eccezionali, non ricorrenti nel caso di specie, di cui all'art. 3 della legge $1^{\circ}$ dicembre 1970, n. 898 ("Disciplina dei casi di scioglimento del matrimonio"), solo se preceduto da una declaratoria di separazione personale ${ }^{24}$.

10. Esaminando il profilo inerente all'individuazione della legge applicabile, il Tribunale ritiene attestata in modo univoco dagli scritti difensivi delle parti la volontà comune di assoggettare la regolazione del divorzio alla propria legge nazionale comune: la moglie, infatti, nella formulazione del ricorso di divorzio aveva espressamente invocato il Codice della famiglia moldavo e, a sua volta, il marito non solo non si era opposto al divorzio, ma aveva altresì chiesto il rigetto della domanda di addebito del divorzio, non essendo tale istituto contemplato nell'ordinamento moldavo. Da ciò, il Tribunale ritiene di poter ricavare in modo inequivocabile il comune intendimento delle parti di vedere applicata, tra quelle testualmente indicate dall'art. 5, par. 1, del regolamento Roma III, la legge nazionale comune indicata alla lett. c), richiamandosi ad un principio consolidato nella prassi applicativa, quello cioè secondo il quale non è necessario ai fini del perfezionamento dell'optio legis che le dichiarazioni dei coniugi siano contestuali e raccolte in un unico documento.

11. Per quanto concerne in particolare il requisito minimo della forma scritta stabilito dall'art. 7, par. 1, del regolamento allo scopo di assicurare che la scelta sia formulata dai coniugi in modo consapevole, esso risulta soddisfatto nel caso di specie dal fatto che la volontà è stata manifestata da entrambi i coniugi rispettivamente nel ricorso e nella memoria di costituzione. Non sono d'altro canto previsti nell'ordinamento italiano requisiti di forma supplementari. Sulla tempestività dell'accordo, il Tribunale rileva come la scelta dei coniugi a favore della legge moldava risulti chiaramente sin dalla fase introduttiva del giudizio (ricorso e memoria di costituzione del resistente), osservando peraltro come nell'ordinamento italiano l'electio juris debba ritenersi ammissibile anche nel corso del procedimento,

\footnotetext{
22 Sul punto v. CASS. CIV., 25 luglio 2006, n. 16978, in Rivista di diritto internazionale privato e processuale, 2007, p. 432.

${ }^{23}$ Il testo integrale della sentenza è reperibile nella Banca dati "Leggi d'Italia" De Agostini.

${ }^{24}$ In particolare, secondo quanto previsto dalla citata legge n. 898/1970, nel testo modificato dalla legge 6 maggio 2015, n. 55 (c.d. "Divorzio breve"), il periodo di separazione deve essersi protratto per un anno, nel caso della separazione giudiziale, o di sei mesi, nel caso di separazione consensuale (art. 3).
} 
purché essa intervenga entro i termini previsti dall'art. $709,3^{\circ}$ comma, cod. proc. civ., e dall'art. $4,5^{\circ}$ comma, della legge n. 898/1970, affinché le parti possano integrare e precisare gli elementi di diritto costituenti le ragioni delle rispettive domande.

12. Il Tribunale accoglie pertanto la domanda di scioglimento del matrimonio in applicazione dell'art. $33,2^{\circ}$ comma, del Codice della famiglia moldavo, ai sensi del quale "il matrimonio può cessare tramite divorzio in base alla richiesta di uno o entrambi i coniugi", riscontrando nel caso di specie l'impossibilità di ricostituire la comunione materiale e spirituale di vita tra i coniugi. Sulla base della medesima legge, il Tribunale respinge la richiesta di addebito formulata dalla moglie, atteso che l'istituto non trova alcuna corrispondenza nell'ordinamento designato.

È questo un profilo sul quale il Tribunale non ritiene utile soffermarsi, ma di rilevante interesse. Sul punto, è appena il caso di notare che l'optio legis deve intendersi diretta ad individuare la legge materiale di un singolo ordinamento statale, senza possibilità di selezionare da una pluralità di ordinamenti le singole disposizioni ritenute più favorevoli rispetto ai propri interessi (c.d. cherry-picking) ${ }^{25}$. Nel caso in esame, peraltro, la richiesta avente ad oggetto l'addebito proviene unilateralmente da una delle parti. La questione, allora, potrebbe essere quella di ritenere non validamente formatosi l'accordo dei coniugi in merito alla lex divortii proprio nella misura in cui nel ricorso introduttivo la moglie evoca contestualmente l'applicazione di una specifica norma dell'ordinamento italiano che non trova alcuna corrispondenza in quello moldavo. Ogni dubbio in tal senso è tuttavia fugato nella fattispecie concreta dalla successiva presentazione da parte dei coniugi, in sede di precisazione delle conclusioni, di un accordo volto specificamente a designare la legge moldava come legge regolatrice del divorzio.

Ne risulta confermata in ogni caso l'importanza di effettuare l'optio legis non solo tempestivamente - nel rispetto dunque dei termini processuali desumibili dalla lex fori - ma anche in termini univoci: una scelta formulata in modo non immediatamente chiaro rischia infatti di riflettersi in danno dei coniugi, dilatando i tempi di definizione del giudizio e comportando un inutile dispendio di attività processuali. Vi è poi il rischio che il giudice reputi invalida la scelta effettuata, ripiegando sui criteri oggettivi stabiliti dall'art. 8 del regolamento: in entrambi i casi sin qui analizzati, ciò avrebbe condotto all'applicazione della legge italiana quale legge dello Stato di residenza abituale dei coniugi (lett. $a$ ) ), con l'effetto di precludere la possibilità di accedere direttamente al divorzio in assenza di una previa separazione, a detrimento degli interessi dei coniugi ${ }^{26}$.

13. Da ultimo, occorre rilevare come nei casi in cui i coniugi si sono avvalsi della facoltà di scegliere la legge applicabile, ciò è avvenuto limitatamente al profilo attinente allo status, secondo quanto previsto dall'art. 5 del regolamento (UE) n. 1259/2010, mentre nulla è stato disposto dagli stessi coniugi in ordine alla legge applicabile alle domande accessorie di volta in volta formulate, in particolare per quanto riguarda le obbligazioni di mantenimento a favore della prole, o di uno dei due coniugi nei confronti dell'altro, ovvero i rapporti patrimoniali tra i coniugi. Si tratta, come noto, di aspetti che esulano dall'ambito di applicazione del regolamento Roma III, da ricondurre ad altri regolamenti - preesistenti o successivamente adottati - dell'Unione europea, i quali parimenti concedono spazio all'esercizio dell'optio legis ${ }^{27}$. Risultano perciò ancora inespresse nella prassi applicativa italiana le potenzialità, pun-

${ }^{25}$ Tale pratica, come noto, risulta preclusa persino nel settore delle obbligazioni contrattuali, tipicamente ispirato alla più ampia valorizzazione della volontà delle parti. Dal regolamento Roma III risulta peraltro esclusa anche la diversa possibilità di operare un dépeçage della fattispecie - quest'ultima, invece, ammessa in ambito contrattuale: v. l'art. 3, par. 1, secondo periodo, del regolamento (CE) n. 593/2008 sulla legge applicabile alle obbligazioni contrattuali, c.d. "Roma I": sul punto, v. P. HAMmJE, "Le nouveau règlement (UE) nº 1259/2010 du Conseil du 20 décembre 2010 mettant en oeuvre une coopération renforcée dans le domaine de la loi applicable au divorce et à la séparation de corps", Revue critique de droit international privé, 2011, p. 291 ss., p. 315.

${ }_{26}$ È rimasta priva di conseguenze pregiudizievoli per i coniugi l'errata valutazione operata da TRIB. CosENZA, 18 settembre 2017, n. 1834, che ha pronunciato il divorzio tra due coniugi albanesi in applicazione della legge nazionale comune - quindi in assenza di un previo periodo di separazione - invocando l'art. 8, lett. c), del regolamento sebbene vi fossero i presupposti per ricavare dagli scritti difensivi che l'applicazione della legge albanese fosse in realtà il frutto della scelta congiunta delle parti.

27 Oltre al già citato regolamento (UE) n. 1103/2016 in tema di regimi patrimoniali tra coniugi, viene in considerazione in particolare il regolamento (UE) n. 4/2009 del Consiglio, del 18 dicembre 2008, relativo alla competenza, alla legge applicabile, al riconoscimento e all'esecuzione delle decisioni e alla cooperazione in materia di obbligazioni alimentari (il quale a sua volta rinvia, attraverso l'art. 15, al Protocollo dell'Aja del 2007), in GUUE L 7 del 10 gennaio 2009, p. 1 ss. 
tualmente evidenziate dalla dottrina, che un accorto esercizio dell'autonomia internazionalprivatistica è in grado di spiegare in un quadro normativo estremamente frammentato, riconducendo ad un'unica legge i molteplici aspetti - distinti l'uno dall'altro, ma al contempo strettamente connessi - che richiedono, con l'insorgere della crisi matrimoniale, di essere regolati attraverso l'intervento del giudice ${ }^{28}$.

\section{La prassi applicativa sull'art. 8 del regolamento (UE) n. 1259/2010: l'individuazione della legge applicabile in caso di mancato accordo tra i coniugi}

14. Non sempre, tuttavia, come accennato, ricorrono i presupposti per un proficuo esercizio dell'autonomia internazionalprivatistica cui, come sottolineato, il regolamento (UE) n. 1259/2010 conferisce rilievo primario. La prassi applicativa evidenzia infatti le difficoltà che si frappongono, specialmente nel contesto di un procedimento di natura contenziosa, alla possibilità di addivenire ad un accordo tra $\mathrm{i}$ coniugi in ordine alla legge applicabile alla separazione personale o al divorzio ${ }^{29}$. Se dunque non può escludersi che in talune ipotesi il mancato esercizio dell'optio legis possa ricondursi ad un comportamento inerte dei coniugi, magari non adeguatamente informati sul punto dai propri difensori, la ragione è da individuarsi nella generalità dei casi nell'impossibilità oggettiva di pervenire di comune accordo alla designazione della legge applicabile, determinata, a seconda dei casi, da un'irriducibile divergenza di vedute tra i coniugi oppure dall'impossibilità materiale di acquisire il consenso dell'altro coniuge, resosi irreperibile: del resto, la scelta della legge applicabile alla separazione o al divorzio non viene normalmente effettuata se non quando la crisi matrimoniale è ormai irrimediabilmente in atto.

In tale scenario, assume rilievo centrale la disciplina posta dall'art. 8 del regolamento, il quale, per le ipotesi di mancato accordo tra le parti, individua la legge regolatrice alla stregua di una sequenza di criteri oggettivi operanti secondo un concorso successivo ${ }^{30}$. Tra questi, un ruolo privilegiato viene accordato alla legge dello Stato di residenza abituale dei coniugi quale legge maggiormente idonea a soddisfare le esigenze di prossimità che permeano l'impianto del regolamento; ove invece i coniugi risiedano in Stati diversi, il criterio di collegamento è individuato nella legge dello Stato di localizzazione dell'ultima residenza abituale comune dei coniugi, alla duplice condizione che non sia trascorso più di un anno dal momento in cui questa è cessata e che uno dei coniugi continui a risiedervi (rispettivamente lett. $a$ ) e lett. $b$ ) ). In via subordinata, la disposizione richiama la legge di comune cittadinanza dei coniugi (lett. $c$ ) ) e, in ultima istanza, la lex fori (lett. $d$ ) $)^{31}$. Come è agevole notare, l'elenco è modellato sulla falsariga dell'art. 5, par. 1, con due importanti precisazioni: per quanto concerne la legge nazionale, si fa riferimento alla comune cittadinanza dei coniugi per l'ovvia esigenza di garantire equidistanza tra i coniugi; inoltre, la sussistenza dei singoli elementi di connessione indicati deve essere accertata avendo riguardo al momento in cui viene adita l'autorità giurisdizionale.

28 Sul valore aggiunto che in tale quadro può spiegare la libera determinazione delle parti, v. P. FRANZINA, "L'autonomia della volontà nel regolamento sui conflitti di leggi in materia di separazione e divorzio", cit., p. 495, che osserva come l'autonomia internazionalprivatistica "si conferma capace di fungere da strumento di razionalizzazione di regimi conflittuali complessi, contro gli inconvenienti insiti nella diversificazione delle fonti e nella specializzazione delle regole di conflitto". In senso analogo, v. R. CLERICI, "Il nuovo regolamento dell'Unione europea sulla legge applicabile al divorzio e alla separazione personale", cit., p. 1064.

29 Per tale rilievo, v. R. CleRICI, "Il nuovo regolamento dell'Unione europea sulla legge applicabile al divorzio e alla separazione personale", cit., p. 1053, che osserva come "una simile autonomia appare soprattutto esercitabile nelle ipotesi di separazione personale o di scioglimento del matrimonio su base consensuale, oltre che nel caso di coppie particolarmente attente e (pessimisticamente) lungimiranti, in grado di pianificare con largo anticipo (o addirittura al momento del matrimonio) la legge applicabile alla futura patologia del loro vincolo, eventualmente in concomitanza con la scelta del regime patrimoniale".

30 Peraltro, l'operatività dell'art. 8 non risulta circoscritta alle ipotesi di mancato esercizio dell'optio legis, estendendosi altresì alle fattispecie in cui la scelta sia stata effettuata dai coniugi senza ottemperare alle condizioni fissate dalla normativa uniforme, nonché, come si ricava dall'art. 12, nel caso in cui l'applicazione della legge straniera designata dalle parti produca effetti manifestamente incompatibili con l'ordine pubblico del foro.

${ }^{31}$ Per un approfondimento sull'art. 8, cfr. J. CARRASCOSA GonzÁLez, "La Ley aplicable a la separación judicial y al divorcio en defecto de elección de ley por los cónyuges. Análisis del artículo 8 del Reglamento 1259/2010 de 20 dicembre 2010", questa Rivista, 2012, p. 52 ss.; Z. Crespi ReghizzI, sub Art. 8, in P. Franzina (a cura di), "Regolamento (UE) n. 1259/2010 del Consiglio del 20 dicembre 2010 relativo all'attuazione di una cooperazione rafforzata nel settore della legge applicabile al divorzio e alla separazione personale. Commentario", Le Nuove leggi civili commentate, 2011, p. 1435 ss., p. 1491 ss. 
15. La vicenda familiare all'origine della pronuncia emessa dal Tribunale di Modena in data 26 gennaio 2016 risulta emblematica delle difficoltà evocate ${ }^{32}$. L'istanza di separazione viene presentata da una cittadina marocchina, unita in matrimonio ad un proprio connazionale, la quale allega l'irreparabile deterioramento del rapporto coniugale a fronte dell'abbandono da parte del marito della casa familiare e del suo ritorno in patria, al quale era poi seguita la totale interruzione di qualsiasi forma di comunicazione anche nei confronti della figlia minore della coppia. Oltre alla domanda di separazione, la donna agisce in giudizio per ottenere l'affidamento della figlia minore e la corresponsione a favore di quest'ultima da parte del marito di un assegno di mantenimento. Il marito, non costituitosi in giudizio sebbene ritualmente citato, viene dichiarato contumace.

16. Per quanto concerne il profilo attinente allo status, dopo aver verificato positivamente la sussistenza della giurisdizione italiana alla stregua del criterio indicato dall'art. 3, par. 1, lett. $a$ ), secondo trattino, del regolamento Bruxelles II-bis (ultima residenza abituale dei coniugi, se uno di essi vi risiede ancora), il Tribunale individua correttamente il parametro normativo di riferimento nell'art. 8 del regolamento (UE) n. 1259/2010, escludendo di conseguenza l'applicazione della disciplina di diritto interno di cui all'art. 31 della legge n. 218/1995, la cui portata risulta ormai circoscritta ai soli procedimenti avviati prima della data di applicazione del regolamento, ossia prima del 21 giugno 2012.

I criteri di collegamento oggettivi previsti dalla disciplina uniforme vengono rapidamente passati in rassegna dal Tribunale per scartarne, in sequenza, l'applicazione: in mancanza di una residenza abituale comune, e dovendosi parimenti escludere l'applicazione del criterio dell'ultima residenza abituale comune - essendo trascorso oltre un anno dal trasferimento all'estero del marito - il Tribunale ritiene di non poter applicare nemmeno il criterio della comune cittadinanza dei coniugi: ciò in quanto il diritto privato marocchino non contempla l'istituto della separazione personale che costituisce l'oggetto specifico della domanda formulata in giudizio dalla ricorrente. Il Tribunale ripiega pertanto sull'applicazione della lex fori, che, come già ricordato, nella gerarchia dei criteri individuata dall'art. 8 si colloca all'ultimo posto (lett. $d$ ) ), come criterio destinato ad entrare in gioco soltanto ove risulti preclusa la possibilità di applicare quelli previsti con ordine prioritario, pronunciando la separazione personale dei coniugi sulla base dell'art. $151,1^{\circ}$ comma, cod. civ.

17. Nel giungere a tale esito applicativo, in sé corretto, il Tribunale tralascia peraltro di individuarne il fondamento, che pare potersi rinvenire nell'art. 10 del regolamento (UE) n. 1259/2010. Ispirandosi a considerazioni materiali, la disposizione ora richiamata impone l'applicazione della legge del foro nelle ipotesi in cui la legge applicabile individuata in applicazione delle norme di conflitto uniformi stabilite dallo stesso regolamento non preveda l'istituto del divorzio ${ }^{33}$. Nonostante il silenzio della norma, che testualmente si riferisce soltanto alla mancata previsione del divorzio, sembra lecito ritenere che la medesima soluzione possa estendersi alle ipotesi in cui sia invece il diverso istituto della separazione personale a non essere contemplato nell'ordinamento richiamato ${ }^{34}$.

\footnotetext{
32 Il testo integrale della sentenza è pubblicato sulla Banca dati "Leggi d'Italia", De Agostini.

${ }^{33}$ La soluzione accolta dal regolamento si pone, sotto tale aspetto, in linea di continuità con la disciplina di conflitto posta dall'art. 31, $2^{\circ}$ comma, della legge n. 218/1995, contribuendo ad enucleare un vero e proprio diritto al divorzio, a prescindere dalla cittadinanza del soggetto: per tale rilievo, v. B. NASCIMBENE, "Divorzio e diritto internazionale privato", in R. BARATTA (a cura di), Diritto internazionale privato. Dizionari del diritto privato promossi da Natalino Irti, Milano, Giuffrè, 2011, p. 156 ss., p. 161. La stretta inerenza delle situazioni familiari alla sfera esistenziale e personale dell'individuo è, d'altro canto, oggetto di unanime riconoscimento: così espressamente Guido AlPa, Elena Bargelli, "Premessa: i rimedi alla crisi familiare", in G. Ferrando, M. Fortino, F. Ruscello (a cura di), Famiglia e Matrimonio, vol. I, tomo II, del Trattato di diritto di famiglia diretto da Paolo Zatti, Milano, Giuffrè, p. 1223 ss., p. 1288. L'art. 10 del regolamento Roma III si applica, inoltre, alle ipotesi in cui la lex causae discrimini sulla base del sesso il diritto dei coniugi di accedere al divorzio, imponendo a sfavore di uno di essi il rispetto di condizioni più restrittive: il riferimento è in particolare al ripudio unilaterale previsto a favore esclusivo del marito.

${ }^{34}$ Così A. Leandro, sub Art. 10, in P. Franzina (a cura di), "Regolamento (UE) n. 1259/2010 del Consiglio del 20 dicembre 2010 ...”, cit., p. 1503 ss., p. 1506. Per l'opinione contraria, v. J. CARrascosa GonZÁlez, "Esclusione dell'applicazione della legge regolatrice del divorzio e regolamento Roma III", cit., p. 275 s., che puntualizza che, se l'ordinamento richiamato non prevede la separazione personale, ma ammette la possibilità di addivenire allo scioglimento del vincolo, il giudice adito non potrà in questo caso ripiegare sull'applicazione della legge del foro, ma dovrà respingere la domanda di separazione personale.
} 
A tale stregua, l'art. 10 isola uno specifico aspetto attinente alla salvaguardia dell'ordine pubblico interno, che trova considerazione in termini più ampi nella clausola di cui all'art. $12^{35}$. Sembra utile ricordare, sotto tale profilo, che la compatibilità con l'ordine pubblico interno degli effetti dell'applicazione in Italia della legge marocchina era già stata vagliata di fronte alle corti nazionali, ma da un'altra angolazione: in relazione ad istanze di divorzio sottoposte a tale legge - vuoi attraverso l'optio legis, vuoi in applicazione del criterio oggettivo indicato dall'art. 8, lett. c), del regolamento Roma III - era stato escluso che potesse ravvisarsi un contrasto in ragione della possibilità, prevista nell'ordinamento marocchino, di accedere al divorzio per mutuo consenso senza un periodo di previa separazione ${ }^{36}$.

Nel caso di specie, invece, il contrasto viene rilevato dall'organo giudicante in quanto l'istanza avanzata dalla ricorrente ha ad oggetto specificamente la separazione, un istituto non contemplato dalla lex causae. Da questo punto di vista, può osservarsi come l'irreperibilità del marito abbia precluso alla ricorrente di accedere direttamente al divorzio: ne è derivata invero l'impossibilità di raggiungere un accordo sulla legge applicabile e di selezionare, per questa via, la legge nazionale comune come legge applicabile sulla base della facoltà offerta dall'art. 5, lett. c), del regolamento: ciò ad evidente riprova di quanto pervasivi possano essere i riflessi che, in funzione della legge applicabile, si producono sul tipo di istanza formulabile in giudizio e di conseguenza sulla tutela dei diritti dei singoli ${ }^{37}$.

18. La lex fori, si noti, viene applicata dal Tribunale di Modena anche rispetto alle altre istanze formulate dalla ricorrente, aventi ad oggetto l'affidamento della figlia minore e la richiesta di un contributo al mantenimento della stessa: è tuttavia omesso qualsiasi cenno alle pertinenti norme di conflitto.

Si tratta di una tendenza in effetti piuttosto diffusa nella prassi, che riflette la difficoltà dell'interprete di orientarsi in un quadro normativo - come già si è avuto modo di notare - assai articolato e complesso, in base al quale i diversi aspetti di una vicenda complessa come quella della crisi matrimoniale sono da ricondurre a strumenti normativi distinti, di derivazione sovranazionale, che hanno progressivamente eroso l'originario ambito di applicazione della disciplina di conflitto interna di cui alla legge n. $218 / 1995^{38}$. Resta peraltro da segnalare che, con la riforma del diritto della filiazione operata nel 2012, è stato inserito nella legge n. 218/1995 un nuovo articolo, l'art. 36-bis, che qualifica come di applicazione necessaria, rendendole dunque applicabili nonostante il richiamo ad altra legge, "le norme

35 In base all'art. 12 del regolamento, “[1]'applicazione di una norma della legge designata in virtù del presente regolamento può essere esclusa solo qualora tale applicazione risulti manifestamente incompatibile con l'ordine pubblico del foro". Ove la lex causae venga disapplicata per asserito contrasto con l'ordine pubblico, il diritto applicabile dovrà essere individuato secondo i criteri propri di ciascun ordinamento: v. per quanto riguarda l'Italia l'art. 16, $2^{\circ}$ comma, della legge n. 218/1995, secondo il quale il giudice dovrà applicare la legge richiamata mediante altri criteri di collegamento eventualmente previsti per la medesima ipotesi normativa ovvero, in mancanza, la legge italiana.

${ }^{36}$ In tal senso, v. TRIB. Belluno, 27 ottobre 2016, supra, par. 4.

37 Sul punto, v. anche Trib. Mantova, 19 gennaio 2016, che ha dichiarato inammissibile la domanda di divorzio, e le ulteriori domande connesse, avanzate da una cittadina cinese nei confronti del marito, anch'egli di nazionalità cinese, sulla base della legge italiana - applicabile ex art. 8, lett. b) del regolamento (UE) n. 1259/2010 - posto che la legge italiana, a differenza di quella cinese, pur invocata dalla ricorrente, non ammette lo scioglimento del matrimonio senza una previa declaratoria di separazione personale. Il marito risultava irreperibile e per questo non era stato possibile acquisirne il consenso per designare di comune accordo la legge cinese ed ottenere, per tale via, il divorzio immediato. Sottolinea come, in caso di mancato accordo tra le parti, le norme uniformi conducano in molti casi a precludere l'accesso al divorzio immediato previsto da diversi ordinamenti, con riferimento specifico alla legge marocchina, R. CLERICI, "Il ruolo dell'autonomia privata tra espansione e limiti di operatività nel regolamento (CE) n. 1259/2010 ...”, cit., p. 359.

38 In relazione ad alcuni aspetti, invece, le norme di conflitto interne conservano la propria efficacia: si pensi ad esempio alle condizioni per contrarre matrimonio (art. 27) e alla forma del matrimonio (art. 28). Diverse pronunce affrontano in particolare il profilo della mancata trascrizione nei registri dello stato civile italiano del matrimonio celebrato all'estero (rispetto al quale trovano applicazione le disposizioni dell'Ordinamento dello stato civile contenute nel D.P.R. 3 novembre 2000, n. 396), escludendo che ciò costituisca una condizione ostativa rispetto alla statuizione sul vincolo da parte della corti italiane, in linea con il principio, affermato a più riprese dalla giurisprudenza di legittimità, che attribuisce alla trascrizione del matrimonio nell'ordinamento italiano un'efficacia non già costitutiva, ma meramente dichiarativa (cfr. CASS. CIV., Sez. un., 28 ottobre 1985, n. 5292, in Rivista di diritto internazionale privato e processuale, 1987, p. 92 ss.). Dovrà nondimeno accertarsi, in applicazione dell'art. 28 della legge n. 218/1995, il rispetto dei requisiti di forma richiesti, alternativamente, dalla legge del luogo di celebrazione del matrimonio, dalla legge nazionale di almeno uno dei coniugi ovvero dalla legge dello Stato di comune residenza dei coniugi al momento della celebrazione del matrimonio: sul punto, v. ex multis Trib. PArma, 2 gennaio 2017; Trib. Roma, 19 maggio 2017. 
del diritto italiano che $a$ ) attribuiscono ad entrambi i genitori la responsabilità genitoriale; $b$ ) stabiliscono il dovere di entrambi i genitori di provvedere al mantenimento del figlio; $c$ ) attribuiscono al giudice il potere di adottare provvedimenti limitativi o ablativi della responsabilità genitoriale in presenza di condotte pregiudizievoli per il figlio" 39 .

19. Sono molteplici le sentenze che, analogamente, hanno pronunciato la separazione personale o il divorzio tra i coniugi sulla base della legge italiana quale legge dello Stato di residenza abituale dei coniugi oppure quale legge dell'ultima residenza abituale della coppia, come previsto dall'art. 8 del regolamento, rispettivamente lett. $a$ ) e lett. $b)^{40}$. In altri casi, più sporadici, 1'applicazione della legge italiana risulta dal criterio residuale stabilito dall'art. 8, lett. $d$ ), ossia a titolo di legge dello Stato del foro ${ }^{41}$. Nel complesso, non risultano particolari difficoltà applicative, anche se talvolta la giurisprudenza di merito omette di indicare il singolo criterio rilevante nel caso concreto. Di fatto, accade frequentemente che i criteri oggettivi indicati dall'art. 8 del regolamento, per le ipotesi di mancato esercizio dell'optio legis da parte dei coniugi, conducano al medesimo esito applicativo ${ }^{42}$. Ne deriva una corrispondenza tra forum e jus che risulta indubbiamente proficua, per le ragioni già evidenziate in termini di semplificazione e di efficienza processuale e che, tuttavia, non appare priva di riflessi negativi per i coniugi, ove la legge nazionale di questi ultimi consenta di accedere più facilmente allo scioglimento del vincolo.

20. Frequenti sono i casi in cui l'istanza di separazione è stata formulata in giudizio con contestuale richiesta di addebito a carico del coniuge, per lo più rimasto contumace nel procedimento ${ }^{43}$. Nella generalità dei casi, anche quando la richiesta di addebito ha trovato accoglimento - in particolare allorché sono stati riscontrati comportamenti violenti del marito nei confronti della moglie e dei figli -e non è stata invece rigettata per mancanza di prove, le corti interne hanno trascurato di inquadrare giuridicamente tale istituto attraverso il riferimento alle pertinenti norme di conflitto, applicando de plano la legge italiana ${ }^{44}$. Dalle pronunce esaminate sembra comunque trasparire l'inclinazione dei giudici italiani a qualificare la questione dell'addebito come ancillare a quella relativa allo status, riconducendola

39 Cfr. la legge 10 dicembre 2012, n. 219 ("Disposizioni in materia di riconoscimento dei figli naturali") e il d.lgs. 28 dicembre 2013, n. 154 ("Revisione delle disposizioni vigenti in materia di filiazione, a norma dell'articolo 2 della legge 10 dicembre 2012, n. 219").

${ }^{40}$ Hanno pronunciato la separazione personale o il divorzio sulla base della legge italiana in applicazione dell'art. 8, lett. a) del regolamento Roma III, TRIB. RoMA, 26 gennaio 2016, n. 1584 (separazione tra coniugi di nazionalità straniera, non specificata); TRIB. Roma, $1^{\circ}$ giugno 2016 (separazione tra coniugi di nazionalità filippina); TRIB. RomA, 28 giugno 2016 (separazione tra un cittadino colombiano nei confronti della moglie ecuadoriana); TRIB. RomA, 5 agosto 2016 (separazione tra una cittadina italiana e un cittadino egiziano); TRIB. RomA, 8 agosto 2016 (istanza di separazione avanzata in giudizio da un cittadino italotunisino nei confronti della moglie rumena); TRIB. RoMA, 14 ottobre 2016 (separazione tra un cittadino italiano e la moglie cubana); TRIB. Roma, 11 ottobre 2016 (istanza di separazione introdotta da una cittadina italiana nei confronti del marito tunisino); TRIB. Roma, 28 settembre 2016, n. 17955 (separazione tra una cittadina italiana nei confronti del marito marocchino); TRIB. Roma, 8 marzo 2016, n. 4804 (separazione tra una cittadina italiana e un cittadino polacco); TRIB. Roma, 14 ottobre 2016 (separazione tra una cittadina italiana e un cittadino marocchino); TRIB. RomA, 18 novembre 2016 (separazione tra una cittadina italiana e un cittadino cinese); TRIB. RoMA; 12 dicembre 2016 (separazione tra una cittadina italiana e un cittadino nigeriano); TRIB. Roma, 9 agosto 2016 (divorzio tra due cittadini peruviani); TRIB. RomA, 16 giugno 2017 (divorzio tra una cittadina peruviana e un cittadino italiano). Hanno pronunciato la separazione, sempre in applicazione della legge italiana, avendo riguardo però all'ultima residenza abituale dei coniugi, come previsto dall'art. 8 , lett. $b$ ), del regolamento Roma III, TRIB. AOSTA, 10 luglio 2017 (istanza di separazione presentata dal marito italo-marocchino nei confronti della moglie marocchina); TRIB. RoMA, 17 marzo 2017 (separazione tra una cittadina peruviana e un cittadino italiano); TRIB. RoMA, 21 aprile 2017 (separazione tra un cittadino italo-brasiliano e una cittadina di nazionalità statunitense).

${ }^{41}$ Cfr. TRIB. Roma, 13 settembre 2016 (separazione richiesta da un cittadino italiano nei confronti della moglie ruandese); Trib. di Roma, 19 maggio 2017 (separazione con addebito richiesta da una cittadina nei confronti del marito tedesco).

42 V. TRIB. PADOva, 3 marzo 2017, che, in relazione all'istanza di divorzio presentata da una cittadina marocchina nei confronti del marito italiano, ha applicato la legge italiana invocando non già la lett. $a$ ), come sarebbe stato corretto, dato che entrambi i coniugi avevano mantenuto la propria residenza abituale in Italia, ma la lett. $d$ ), che fa riferimento diretto alla lex fori.

43 In argomento, v. I. Aquironi, L'addebito della separazione nel diritto internazionale privato dell'Unione europea, questa Rivista, 2017, p. 76 ss.

${ }^{44}$ Viene in rilievo in particolare l'art. $151,2^{\circ}$ comma, cod. civ., secondo il quale "[i]l giudice, pronunziando la separazione, dichiara, ove ne ricorrano le circostanze e ne sia richiesto, a quale dei coniugi sia addebitabile la separazione, in considerazione del suo comportamento contrario ai doveri che derivano dal matrimonio". 
pertanto al regolamento Bruxelles II-bis, per quanto riguarda il profilo dell'individuazione del giudice competente, e al regolamento Roma III per quanto concerne invece il diritto applicabile ${ }^{45}$.

21. La sentenza emessa dal Tribunale di Belluno in data 13 giugno 2017 si segnala per aver compiuto un appropriato inquadramento dell'istituto dell'addebito ${ }^{46}$. La richiesta di separazione con addebito viene formulata in giudizio da una cittadina di nazionalità albanese nei confronti del marito, anch'egli di nazionalità albanese. Sul punto, il Tribunale osserva come la richiesta di addebito, pur costituendo una domanda autonoma e eventuale, "risulti inscindibilmente connessa alla domanda di separazione personale, tanto da non poter essere proposta in un diverso giudizio". Sulla base di tale rilievo, il Tribunale esclude che la richiesta di addebito possa essere assoggettata - come in effetti parte della giurisprudenza ritiene ${ }^{47}$ - ad una disciplina diversa da quella prevista rispettivamente dai regolamenti Bruxelles II-bis e Roma III.

Anche in questo caso, il marito è rimasto contumace e non risulta agli atti un preventivo accordo tra le parti in merito alla legge applicabile, con conseguente applicazione dell'art. 8, lett. $b$ ), del regolamento in ultimo richiamato, dovendosi individuare in Italia il luogo dell'ultima residenza abituale dei coniugi nell'ultimo anno che precede l'introduzione del giudizio. Il Tribunale giunge così a pronunciare la separazione personale tra i coniugi sulla base della legge italiana, dopo aver constatato, sulla base di quanto richiesto dall'art. $151,1^{\circ}$ comma, cod. civ., l'irrimediabile deterioramento del rapporto coniugale e l'impossibilità di ripristinare l'ormai cessata convivenza. Circa l'ulteriore istanza formulata dalla moglie, il Tribunale ritiene che, alla luce della condotta violenta del marito - attestata da una condanna penale - vi siano i presupposti per una dichiarazione di addebito a carico del marito (art. $151,2^{\circ}$ comma, cod. civ.).

22. A dimostrazione di come la prassi interna non sia sempre rigorosa nell'applicazione delle norme di diritto internazionale privato di matrice europea, può notarsi, infine, come alcune pronunce applichino la legge italiana alla questione relativa all'assegnazione della casa familiare senza dare conto delle norme di conflitto rilevanti nel caso di specie: la tendenza in questo caso sembrerebbe quella di considerare il provvedimento come misura di protezione del minore, in quanto tale riconducibile, $\mathrm{ra}$ tione temporis, alla Convenzione dell'Aja del 1961 sulla competenza delle autorità e sulla legge applicabile in materia di protezione dei minori, cui l'art. 42 della legge n. 218/1995 rinvia "in ogni caso"48. In generale, poi, il riferimento alla disciplina posta dall'art. 31 della legge n. 218/1995, invero piuttosto ricorrente nella prassi, appare improprio stante il carattere universale o erga omnes che, come già evidenziato, contraddistingue le norme di conflitto dettate dal regolamento (art. 4), dal quale discende

45 Così, ad esempio, ha accolto la richiesta di addebito sulla base della legge italiana, su richiesta avanzata da una cittadina italo-serba nei confronti del marito, di nazionalità kosovara, TRIB. PARMA, 2 gennaio 2017, in Banca Dati "Leggi d'Italia", De Agostini. In senso analogo v. TRIB. PADOVA, 9 maggio 2017 (separazione con addebito, richiesta da una cittadina rumena nei confronti del coniuge, suo connazionale). V. anche TRIB. RomA, 7 luglio 2016, n. 13753 (separazione richiesta da una cittadina italo-francese nei confronti del marito rumeno, accolta con addebito congiunto). Tra le pronunce che, invece, hanno respinto la richiesta di addebito, cfr. TRIB. RoMA, 17 marzo 2017 (separazione tra una cittadina peruviana nei confronti del marito italiano); TRIB. RomA, 21 aprile 2017 (separazione tra un cittadino italo-brasiliano nei confronti della moglie di nazionalità statunitense); TRIB. Roma, 20 gennaio 2017 (separazione tra due coniugi peruviani); TRIB. PADOVA, 13 gennaio 2017 (separazione tra una cittadina ucraina nei confronti del marito italiano).

${ }^{46}$ La sentenza risulta allo stato attuale inedita. In senso conforme, v. TRIB. Belluno, 30 dicembre 2011, in Rivista di diritto internazionale privato processuale, 2012, p. 452.

47 In questi termini, v. Trib. TIVoli, 6 aprile, 2011, in Rivista di diritto internazionale privato processuale, 2011, p. 1097, che assimila la domanda di addebito ad un'azione di risarcimento danni, riconducendola, per quanto concerne la giurisdizione, all'art. 5, n. 3, del regolamento (CE) n. 44/2001 (Bruxelles I), cui corrisponde l'attuale art. 7, n. 2, del regolamento (UE) n. 1215/2012 (Bruxelles I-bis).

48 In tal senso, v. Trib. Roma, 26 gennaio 2016; Trib. Roma, 17 marzo 2017, cit. Ciò in linea con l'art. 337-sexies, cod. civ., introdotto dal d.lgs. 28 dicembre 2013, n. 154, secondo il quale "[i]l godimento della casa familiare è attribuito tenendo prioritariamente conto dell'interesse dei figli”. La Convenzione dell'Aja del 1996 sulla competenza, la legge applicabile, il riconoscimento, l'esecuzione e la cooperazione in materia di responsabilità genitoriale e di misure di protezione dei minori, ratificata ed eseguita dall'Italia con la legge 18 giugno 2015, n. 101, trova applicazione solo con riferimento alle controversie instaurate a decorrere dal $1^{\circ}$ gennaio 2016. 
l'integrale disapplicazione delle corrispondenti norme di conflitto nazionali degli Stati membri che partecipano alla cooperazione rafforzata ${ }^{49}$.

\section{Osservazioni conclusive}

23. A conclusione di questa breve rassegna, può osservarsi come, in relazione alle istanze di divorzio o di separazione personale formulate innanzi ai giudici italiani nelle situazioni di crisi coniugale connotate in senso transnazionale, l'esito applicativo più frequente sia l'applicazione della legge italiana a titolo di legge dello Stato di residenza abituale ovvero di ultima residenza abituale dei coniugi, in applicazione dell'art. 8, lett. $a$ ) e lett. $b$ ), del regolamento (UE) n. 1259/2010. Questo risultato può ritenersi apprezzabile almeno su due versanti: da un lato, sono noti i vantaggi, in primis di ordine pratico, che discendono da una situazione di corrispondenza tra il giudice compente a decidere la controversia e il diritto materiale che questi applicherà; a ciò si aggiungono considerazioni che attengono all'esigenza di favorire l'integrazione dei cittadini stranieri - in questo caso della coppia - nel tessuto sociale dello Stato nel quale essi hanno scelto di stabilire il centro principale dei propri interessi. Accordando alla legge dello Stato di residenza abituale dei coniugi un ruolo privilegiato, tra i criteri oggettivi destinati ad operare in caso di mancata scelta, l'art. 8 del regolamento appare orientato, almeno in parte, in tale direzione.

Vi sono ragioni, d'altro canto, che possono indurre a ritenere insoddisfacente un simile esito applicativo: in molti casi, infatti, ciò determinerà l'impossibilità per la coppia straniera di accedere direttamente al divorzio - ossia senza previa separazione - ove ciò sia previsto dalla legge nazionale comune, in evidente contrasto con uno degli scopi che animano la disciplina uniforme, la quale, raccordandosi al sistema di competenza stabilito dal regolamento Bruxelles II-bis, intende agevolare lo scioglimento del vincolo coniugale (favor divortii) con il proposito di evitare ostacoli all'esercizio delle libertà di circolazione. Da questo punto di vista, si è avuto modo di sottolineare come in effetti la legge italiana, nonostante le novità introdotte dalla legge 6 maggio 2015, n. 55 (c.d. "Divorzio breve"), continui a distinguersi per condizioni di accesso al divorzio che risultano comparativamente più rigorose rispetto a quelle previste negli ordinamenti di altri Stati, membri o non membri dell'Unione europea.

In tale scenario, la scelta della legge regolatrice ad opera dei coniugi, nei limiti tracciati dall'art. 5 del regolamento, è chiamata a svolgere un ruolo determinante, sempre che la situazione di conflittualità tra $\mathrm{i}$ coniugi $\mathrm{o}$, come accade di frequente, l'irreperibilità di uno di essi, non ne impedisca di fatto l'esercizio. I coniugi interessati ad addivenire in tempi più rapidi ad una pronuncia di scioglimento di un vincolo che non corrisponde più alla realtà degli affetti e alle proprie esigenze di vita, oppure animati dall'intento di preservare la propria identità culturale, troveranno nella facoltà di optio legis loro concessa dalla disciplina uniforme uno strumento essenziale affinché nel procedimento che si svolge in Italia sia data applicazione alla propria legge nazionale - in ipotesi, anche la legge di cittadinanza di uno solo di essi - in luogo di quella italiana.

49 Ex multis, v. TriB. Aosta, 10 luglio 2017. 